\title{
Automatic Head MRI segmentation combining FCM and VBM
}

\author{
$\mathrm{Nan} \mathrm{He}^{1, \mathrm{a}}$, Jun Liu ${ }^{2, \mathrm{~b}}$, Helei Wu ${ }^{3, \mathrm{c}}$ \\ ${ }^{1}$ College of Information Engineering, Nanchang Hangkong University, Nanchang, 330063, China \\ ${ }^{2}$ College of Information Engineering, Nanchang Hangkong University, Nanchang, 330063, China \\ ${ }^{3}$ College of Information Engineering, Nanchang University, Nanchang, 330063, China \\ aemail: 87435850@qq.com, bemail: liujun@nchu.edu.cn, cemail: wuhelei2000@163.com
}

Keywords: Image segmentation; FCM algorithm; VBM algorithm

\begin{abstract}
In the diagnosis and operation of the brain, there are five kinds of main tissues, such as gray matter, white matter, cerebrospinal fluid, scalp and skull, those need to be segmented from the MRI to sequel accurate three-dimensional head model. Aiming at this problem, this paper proposes a segmentation method combining FCM \& VBM algorithm. After the craniocerebral regions were extracted from the original MRI images by the BET algorithm, the brain regions were subdivided to obtain gray matter, white matter and cerebrospinal fluid by FCM algorithm. Then, the skull, scalp and brain were segmented by VBM segmentation algorithm. And then the smooth and morphological treatment of the separated tissues was carried out. Finally, five kinds of tissues were obtained. Compared with K-means clustering algorithm and morphological segmentation method, it is found that the segmentation algorithm has lower morphological distortion in the case of higher edge gradient.
\end{abstract}

\section{Introduction}

The brain as the most important and sophisticated nerve center of the brain, the brain of a variety of disease detection and brain surgery require high precision, so the head of the various operations before the need for precise pre-analysis and lesion location. For the pre-operative detection, it is very important to get the $3 \mathrm{D}$ model of the head, which is very important for the diagnosis and operation navigation of brain diseases.

At present for the head area imaging technology mainly CT and magnetic resonance imaging (MRI), which because of MRI for soft tissue has a higher resolution often become the first choice for head lesions. However, because of the complexity of the brain tissue and the similarity of gray levels in the MRI images such as cerebrospinal fluid, skull and head cavities, there are still considerable difficulties in segmenting the various tissues from MRI images. From the literature that has been reported in recent years, there are a variety of algorithms for brain MR image segmentation[1; 2].But these algorithms are mostly for specific areas, such as the extraction of the brain or from the extracted brain region is divided into gray matter, white matter or tumor, etc. [3; 4], and for the head MRI image of the overall segmentation, Especially one-time from the original head MRI image segmentation contains the scalp, skull, cerebrospinal fluid, gray matter, white matter and even the head cavity, including a variety of organizations, there is no universal and effective method.In the segmentation algorithm proposed in recent years, the more prominent head MRI segmentation methods are more classic: Dogdas et al [5] using pure mathematical morphology method from the head MRI image segmentation skull model. The method has the advantages of a simple and practical process, but the disadvantage is due to the skull on the division of the larger scale smoothing led to a large skull shape distortion, in addition to the method is difficult to deal with mandibular parts of the skull. Huang et al [6] proposed a voxel-based morphometry (VBM) method for segmentation of cranial magnetic resonance images. Although this method can segment brain tissue and skull, Cavity and other parts. its shortcomings are divided after the existence of hollow cerebrospinal fluid, there are discontinuous points in the skull, the brain is not identified voxel. 
In order to solve and further optimize the segmentation of human head MRI images, this paper uses the segmentation methods of BET algorithm, FCM algorithm and VBM algorithm to segment the target image. From the original MRI images contains gray matter, white matter, cerebrospinal fluid and skull, as well as the head cavity, and finally in the organizational structure of the smooth, morphological processing and other correction operations, in the case of high fidelity of the border is more Clear three-dimensional structure of various organizations.

\section{Brain Extraction Tool}

The first step of the method is to strip the non-brain tissue from the original MRI image, that is to extract the brain region from the original head MRI image. For this part, we use the BET (Brain Extraction Tool) algorithm [7]. The algorithm was proposed by Smith et al. In 2002 and subsequently widely used in brain research. The basic idea of the BET algorithm is to push the contour point to the edge of the brain tissue with larger gradient through the three forces of direction, different size of tension, smooth force and expansion force. In practical applications, we found that BET algorithm for normal brain parenchyma or virtual brain images can be better and faster segmentation results. Therefore, the brain tissue was obtained by stripping non-brain tissue by BET algorithm.

\section{Fuzzy C - Means Algorithm(FCM)}

For the obtained brain tissue, we have to segment it, resulting in gray matter, white matter, cerebrospinal fluid and other tissue models. Considering that the thresholds of these three kinds of organizations may change within a certain range in different imaging processes, it is difficult to segment with a fixed threshold. So in this paper, we adopt fuzzy C-means algorithm.

The fuzzy clustering algorithm is a kind of clustering algorithm based on the optimal cost function. Fuzzy C-means algorithm is a classical algorithm in the fuzzy clustering algorithm. Fuzzy C-means algorithm [8] proposed by Dunn in 1973, the algorithm is based on the objective function of fuzzy clustering algorithm, the objective function is as follows:

$$
J_{F C M}=\sum_{i=1}^{c} \sum_{j=1}^{n}\left(u_{i j}\right)^{m} d^{2}\left(x_{j}, v_{i}\right) \text { s.t } U \in M_{f}
$$

The fuzzy mean clustering algorithm minimizes the objective function by the Lagrangian multiplier method. The Lagrangian multiplier $\lambda$ is introduced into the objective function to obtain a new function:

$$
L\left(u_{i j}, v_{i}, \lambda_{j}\right)=\sum_{i=1}^{c} \sum_{j=1}^{n}\left(u_{i j}\right)^{m} d^{2}\left(x_{j}, v_{i}\right)+\sum_{j=1}^{n} \lambda_{j}\left(1-\sum_{i=1}^{c} u_{i j}\right)
$$

The partial derivatives of $v_{i}, u_{i j}$ and $\lambda_{i}$ can be obtained for the function $L$, and the derivation of the partial derivatives can be used to derive the cluster center and the update formula of its fuzzy membership degree , which is:

$$
\begin{gathered}
v_{i}=\frac{\sum_{j=1}^{n} u_{i j}^{m} x_{j}}{\sum_{j=1}^{n} u_{i j}^{m}} \\
u_{i j}=\frac{1}{\sum_{r=1}^{c}\left[\left(\frac{d\left(x_{j}, v_{i}\right)}{d\left(x_{j}, v_{r}\right)}\right)^{\frac{2}{m-1}}\right]}
\end{gathered}
$$


The brain tissue was separated by fuzzy C-means algorithm, and the tissues including white matter, cerebral gray matter and cerebrospinal fluid were obtained.

\section{Voxel-based morphometry(VBM)}

The segmented images of brain tissue are obtained by the above method, but for the segmentation of non-brain tissue, the image segmentation of the gray and similar regions is similar due to the similarity of the images of the hollow and skull, and the fuzzy clustering algorithm is less effective for gray similar regions. Brain tissue segmentation, this paper uses VBM segmentation algorithm to get the skull, scalp, cavity and other organizations.

Wright, who in 1995 proposed the "voxel-based MR structure image analysis" of the idea. In 2000, Friston and Ashburner, etc. to Wright and other ideas put forward to improve and summarize, and then propose a new algorithm, that is, VBM algorithm [9]. In this algorithm, Ashburner and other details of the process of dealing with brain images of the steps. The basic steps of the VBM algorithm are to standardize the individual MRI images, that is, to place the individual samples into the same standard space, then segment the MRI images after space normalization, and then smoothen the obtained tissues, Signal to noise ratio, and finally statistical analysis.

In the segmentation process of VBM algorithm, in order to ensure the accuracy of segmentation, it is necessary to make the best registration of the individual MRI images and template images. In 2007, Ashburner et al. [10] proposed the DARTEL algorithm, which uses the flow field theory to perform non-linear registration of the individual images of the sample. The result of the registration is ideal.

VBM algorithm is mainly used in SPM (statistical parametrical mapping) software. The rapid development of VBM algorithm makes the SPM software also increase a lot of functions to improve the accuracy of VBM analysis. In the SPM5 increased DARTEL toolbox, to improve the accuracy of VBM algorithm. In the SPM8 New Segment toolbox for image segmentation, the main use of uniform segmentation method [11]. In the combination of DARTEL Toolbox and New Segment toolbox image segmentation can be better segmentation results. In this paper, SPM8 using DARTEL and New Segment toolbox combined VBM method.

In the process of segmentation, the selection of tissue probability map will greatly affect the effect of segmentation, and the segmentation effect of tissue probability map in New Segment toolbox is not particularly ideal. Therefore, this paper uses CABI (Center for Advanced Brain Imaging ), and the improved tissue prioritization (TPM). The DARTEL algorithm is used to register the individual MRI images of the samples. After registration, the normalized images are normalized, then the normalized images are segmented, and then the tissue is smoothed. Then the statistical analysis of each tissue is carried out. By this method, the non-brain tissue images were divided to obtain the skull, scalp, cavity and other tissues.

After segmenting the brain MRI images, the brain tissue images were segmented by fuzzy C-means algorithm (FCM) and the non-brain tissue images were segmented by VBM algorithm. Finally, the brain MR images were segmented, to obtain a more accurate tissue model.

\section{Algorithm steps}

In summary, the overall MRI image segmentation step can be summarized as follows:

Step 1 Using the BET algorithm to segment the craniocerebral and non-craniocephalic regions from the original MRI images;

Step 2 The FCM algorithm was used to segment the extracted brain regions to obtain gray matter, white matter and cerebrospinal fluid;

Step 3 Using VBM algorithm to segment the non-brain tissue image, get skull, cavity, scalp tissue, and skull smoothing. 


\section{Analysis of results}

In this paper, we selected 63 layers of $256 \times 256$ pixels human head MRI images of the segmentation method was tested, the image gray level of 256.

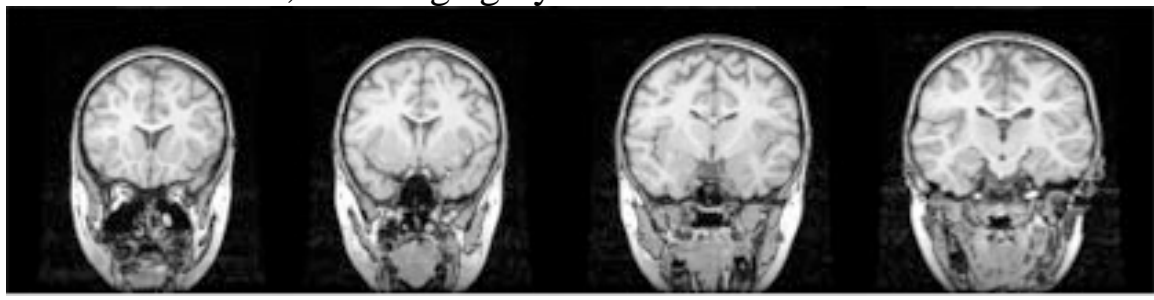

Fig.1 Original MRI image

Figure1 shows the original MRI image. Figure2 shown by BET algorithm extracted from the brain region and non-brain areas:

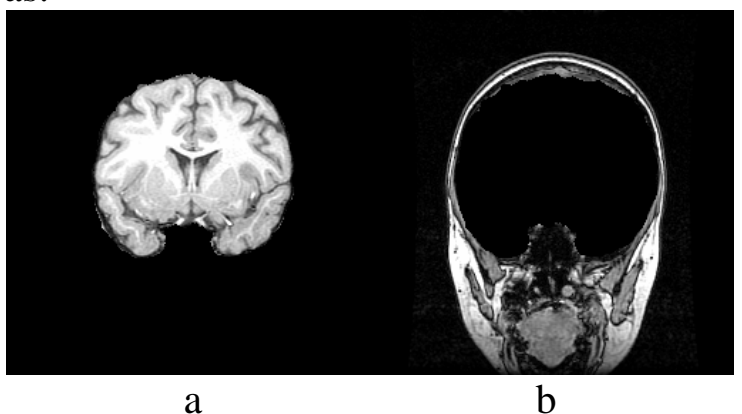

Fig.2 BET segmentation effect. (A) brain regions extracted using the BET algorithm; and (b) residual non-brain tissue.

From Fig.2 can be seen that the brain tissue and non-brain tissue separation effect is obvious, can be more accurate brain tissue and non-brain tissue. Three-dimensional images of the brain areas can be generated in the MRICRO software.

It can be seen that the structure of the brain tissue is complex and the edge of various tissues has some fuzziness, and it is difficult to segment the same fixed threshold for different MRI images. (2), So this paper adopted the FCM algorithm to extract the brain region to be re-segmentation of gray matter, white matter and cerebrospinal fluid three organizations.In addition, we compare the FCM algorithm with the commonly used KM algorithm in the segmentation effect.

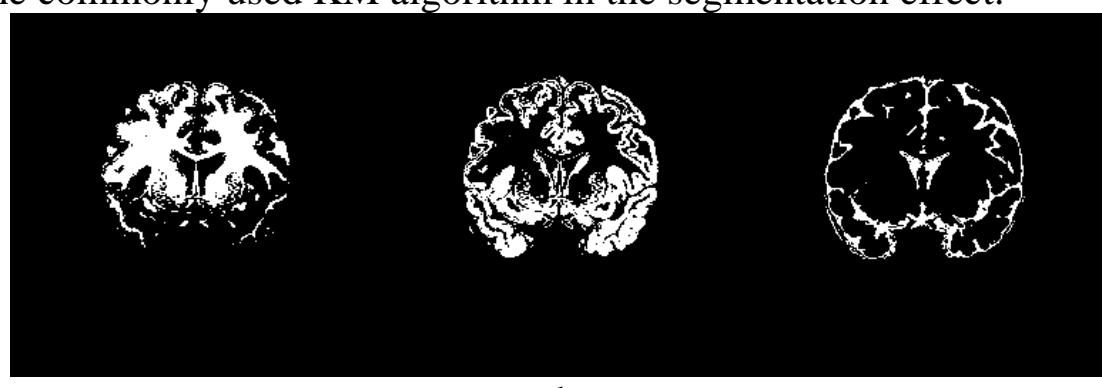

a

b

C

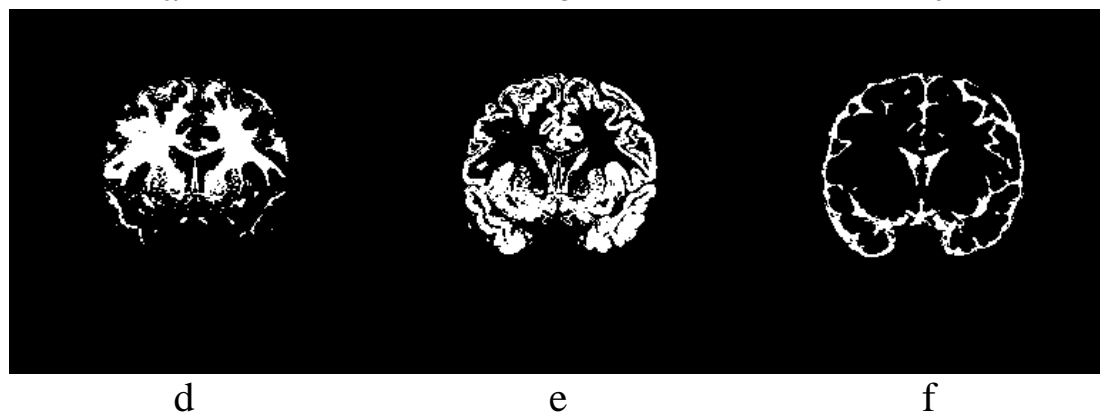

Fig.3 abc for FCM segmentation of brain tissue images; def for the KM after segmentation of brain tissue images. (A) white matter; (b) gray matter; (c) cerebrospinal fluid; (d) white matter; (e) gray matter;(F) Cerebrospinal fluid 
Table (1) FCM algorithm and KM algorithm segmentation of the average image gradient

\begin{tabular}{cccc}
\hline $\begin{array}{c}\text { Segmentation } \\
\text { method }\end{array}$ & $\begin{array}{c}\text { WM image } \\
\text { average } \\
\text { gradient }\end{array}$ & $\begin{array}{c}\text { GM image } \\
\text { average } \\
\text { gradient }\end{array}$ & $\begin{array}{c}\text { CSF image } \\
\text { average } \\
\text { gradient }\end{array}$ \\
\hline FCM & 8.1368 & 13.2369 & 7.5076 \\
KM & 7.2627 & 12.4233 & 6.8628 \\
\hline
\end{tabular}

Fig.3 shows the use of FCM algorithm and KM algorithm were extracted from the brain region to get the gray matter, white matter and cerebrospinal fluid effect. We can see that the two algorithms can get a reasonable segmentation effect, but careful observation can still be found using the KM algorithm of cerebrospinal fluid tissue integrity is poor, there are more intermittent parts, indicating that KM algorithm on the partition threshold search relative FCM algorithm is still a gap. In addition, the edge gradient of FCM algorithm is significantly larger than that of KM algorithm, which indicates that FCM algorithm can separate the tissue image more clearly. Segmentation effect is better.

After the segmentation of the brain tissue by the above method, the VBM algorithm was used to segment the non-brain tissue to obtain the skull, cavity, scalp, and other tissues, because the gray scale of the cavity, skull and part of cerebrospinal fluid were similar. (4) (a) skull image in the figure, there is a certain hole, for building a large impact on three-dimensional model. As a result, the skull image smooth operation, get (4) (d) skull image, 3D Med [12] (4), (e) and (f), the original segmented image can be seen by the noise effect, in the skull by smoothed after the organization. Although the accuracy has decreased, but the skull model of complete form, the effect is good, as shown in Fig.4 (f). Based on the mathematical morphology of the segmentation method to filter the image, morphology and union, morphological operation of the skull and other internal and external boundaries, the skull model obtained by this method is more distorted in morphology and can not deal with the cranial region of the mandible. By comparing the images in (f) and (g), we can see that the segmentation result of this method is more complete and true 


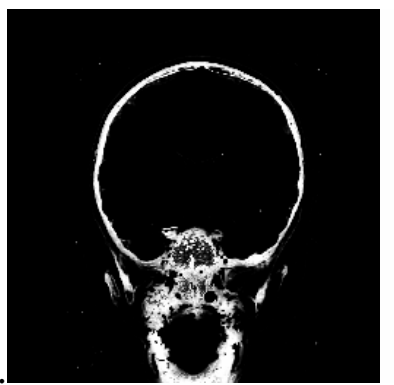

a

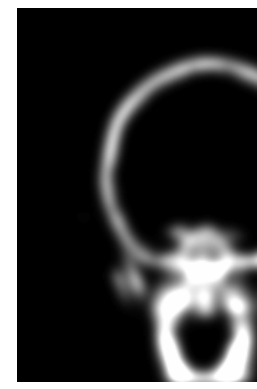

d

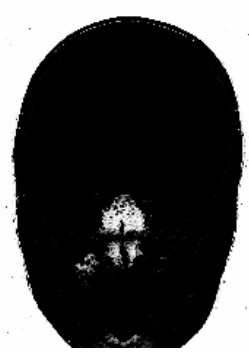

b

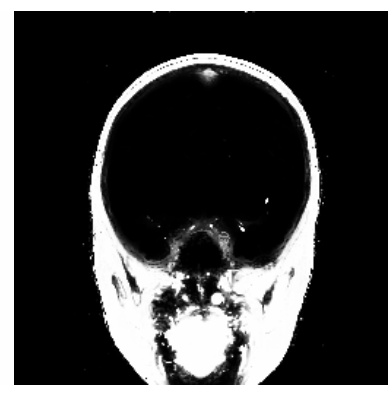

C

e

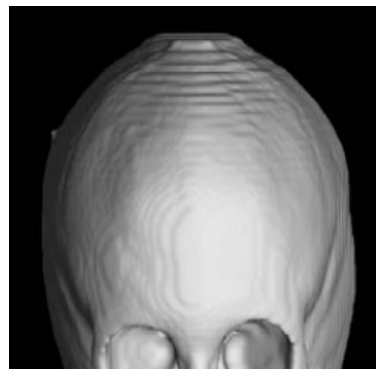

g

Fig.4 VBM algorithm segmentation of non-brain tissue image (a) skull; (b) cavity; (c) scalp;(d) a smooth skull image; (e) a three-dimensional model of the skull after segmentation; (f) a three-dimensional model of the skull after smoothing; (g) a skull model after morphological segmentation.

Fig.5 shows the three-dimensional effect of the above-described five types of head tissues. Although it is difficult to obtain the "gold standard" by medical experts to quantitatively measure the segmentation effect of various organizations, it can be seen that from the morphology of the segmentation of this method can be obtained by the method of the organization of its three-dimensional morphology is reasonable. 

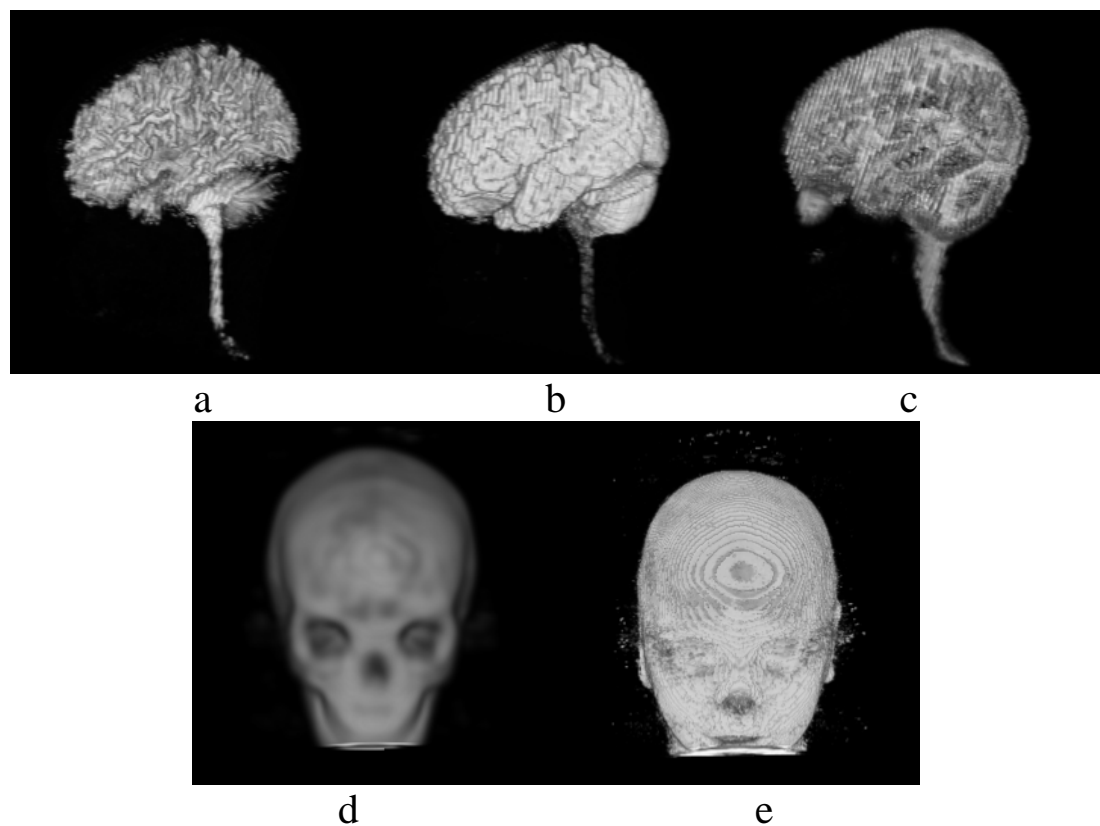

Fig.5 three-dimensional model of the organization.(A) white matter; (b) gray matter; (c) cerebrospinal fluid; (d) skull; (e) scalp

\section{Conclusion}

In this paper, we have successfully segmented the brain MR images by a combination of FCM and VBM. The skull images are smooth, but the skull images are continuous and well-formed. The images of cerebrospinal fluid, white matter segmentation clear. Through the construction of three-dimensional tissue model, we can see that the three-dimensional model is clear, good effect, for the head of the three-dimensional model has a good role. However, the method is complicated, and there are sporadic unidentified regions. Therefore, it is necessary to further study how to simplify the algorithm, normalize the unidentified regions, and modify the segmented images.

\section{Acknowledgement}

In this paper, the research was sponsored by the National Natural Science Fund Project (Project No.61163047) and the Nature Science Foundation of Jiangxi Province (Project No. 20151BAB205050) and Fund Projects In Jiangxi Province Department of Education (Project No. GJJ14503).

\section{References}

[1] Eskildsen S F, Coupé P, Fonov V, et al. BEaST: Brain extraction based on nonlocal segmentation technique[J]. Neuroimage, 2012, 59(3): 2362-73.

[2] Wang Da-xi, Chen Xinxin. Based on the morphology of brain MRI images skull stripping algorithm [J]. Computer technology and development, 2015, (12) : 206-209 + 215.

[3] You Jia-li. Skull brain MR image segmentation algorithm [D]. Graduate school of Chinese academy of sciences (changchun institute of optical precision machinery and physical), 2015

[4] Ma A-min. MRI brain image segmentation and 3D visualization [D]. South China university of technology, 2014.

[5] Dogdas B, Shattuck D W, Leahy R M. Segmentation of the skull in 3D human MR images using mathematical morphology[J]. Proceedings of SPIE - The International Society for Optical Engineering, 2002, 4684: 1553-1562. 
[6] Huang Y, Dmochowski J P, Su Y, et al. Automated MRI segmentation for individualized modeling of current flow in the human head[J]. Journal of Neural Engineering, 2013, 10(6): 358-362.

[7] Smith S M. Fast robust automated brain extraction[J]. Human Brain Mapping, 2002, 17(3): 143-155.

[8] Dunn J C. A Fuzzy Relative of the ISODATA Process and Its Use in Detecting Compact Well-Separated Clusters[J]. Journal of Cybernetics, 1973, 3(3): 32-57.

[9] Ashburner J, Friston K J. Voxel-Based Morphometry-The Methods[J]. Neuroimage, 2000, 11(6 Pt 1): 805-21.

[10] Ashburner J. A fast diffeomorphic image registration algorithm[J]. Neuroimage, 2007, 38(1): 95-113.

[11] Ashburner J, Friston K J. Unified segmentation[J]. Neuroimage, 2005, 26(3): 839-51.

[12] Tian J, Ying Z, Wang J, et al. A 3D Medical Image Processing and Analyzing System[J]. Computerized Tomography Theory \& Applications, 1999. 